\title{
OSCILLATORY INTEGRALS AND SCHRÖDINGER MAXIMAL OPERATORS
}

\author{
LAWrence A. Kolasa
}

\begin{abstract}
In this paper we consider smooth analogues of operators studied in connection with pointwise convergence of the solution of the free Schrödinger equation to the given initial data. Such operators are interesting examples of oscillatory integral operators with degenerate phase functions, and we obtain sharp $L^{2} \rightarrow L^{2}$ bounds.
\end{abstract}

\section{Introduction.}

We begin this paper by giving motivation for the objects we will study, placing them in their proper context.

Consider the initial value problem for the Schrödinger equation with no potential,

$$
\left\{\begin{array}{l}
i \partial_{t} u(x, t)+\Delta_{x} u(x, t)=0 \quad(x, t) \in \mathbb{R}^{n} \times \mathbb{R} \\
u(x, 0)=f(x) \in L^{2}\left(\mathbb{R}^{n}\right) .
\end{array}\right.
$$

Then

$$
u(x, t)=(2 \pi)^{-n} \int_{\mathbb{R}^{n}} e^{i x \cdot \xi} e^{i t|\xi|^{2}} \widehat{f}(\xi) d \xi=\left(e^{i t|\cdot|^{2}} \widehat{f}(\cdot)\right)^{\smile}(x)
$$

defines a (weak) solution of (1) such that $\lim _{t \rightarrow 0} u(x, t)=f(x)$ in the $L^{2}$ sense. When the integral in (2) is absolutely convergent the limit is a pointwise limit. However, if $f$ is an arbitrary $L^{2}$ function the integral in (2) may not be absolutely convergent, and we must take the right hand side of (2) as the definition of $u(x, t)$. It is not self-evident that $u$ converges pointwise to the initial data in this case, and in fact it sometimes does not. The question of what extra smoothness conditions on $f$ will guarantee the existence pointwise a.e. of $\lim _{t \rightarrow 0} u(x, t)$ arises.

For a given $s \geq 0$ let $H^{s}\left(\mathbb{R}^{n}\right)$ denote th e $L^{2}$-Sobolev space,

$$
H^{s}\left(\mathbb{R}^{n}\right)=\left\{f \in L^{2}\left(\mathbb{R}^{n}\right):\|f\|_{H^{s}}=\left(\int_{\mathbb{R}^{n}}\left(1+|\xi|^{2}\right)^{s}|\widehat{f}(\xi)|^{2} d \xi\right)^{1 / 2}<\infty\right\}
$$


In the context of $L^{2}$-Sobolev spaces the question of pointwise convergence to the initial data is completely understood when $n=1$. It was shown by Carleson $[\mathbf{C}]$ that $\lim _{t \rightarrow 0} u(x, t)=f(x)$ whenever $f \in H^{s}(\mathbb{R}), s \geq 1 / 4$. Moreover Dahlberg and Kenig [DK] demonstrated that for all $s<1 / 4$ there are functions $f \in H^{s}(\mathbb{R})$ such that $\varlimsup_{t \rightarrow 0} u(x, t)=\infty$ a.e..

The higher dimensional cases, $n \geq 2$, are not completely understood. For these cases Vega $[\mathbf{V}]$ and Sjölin $[\mathbf{S} \mathbf{j}]$ independently proved that the pointwise limit exists for all $f \in H^{s}\left(\mathbb{R}^{n}\right)$ provided $s>1 / 2$, while there are counterexamples just as in the 1 -dimensional case when $s<1 / 4$. But the question of what happens when $1 / 4 \leq s \leq 1 / 2$ is in general unanswered. However, in $[\mathbf{B}]$, Bourgain shows that there is an $\epsilon>0$ such that $f \in H^{1 / 2-\epsilon}\left(\mathbb{R}^{2}\right)$ guarantees pointwise convergence to the initial data. The value of this $\epsilon$, although in principle calculable, is not given (although $\epsilon \ll 1 / 4$ ). The point here is not what the value of $\epsilon$ is, but that there is some improvement of the above results when $n=2$.

The study of the pointwise behavior of $u(x, t)$ as $t \rightarrow 0$ involves the study of the corresponding maximal operator, the Schrödinger maximal operator,

$$
u^{*}(x)=\sup _{|t| \leq 1}|u(x, t)|
$$

with regard to its mapping properties - i.e., finding weak type or strong type inequalities for $u^{*}$.

The idea in $[\mathbf{B}]$ is to replace the nonlinear operator $u^{*}$ by a family of linear operators. For each measurable function $\mathrm{t}(\mathrm{x})$, defined say on $\mathbb{D}^{n}$, the unit disk in $\mathbb{R}^{n}$, with the property that $|t(x)| \leq 1$, one considers the linear operator

$$
f \longmapsto \int e^{i\left(x \cdot \xi+t(x)|\xi|^{2}\right)} \widehat{f}(\xi) d \xi=u(x, t(x)),
$$

and shows that for some constant $C$, which is independent of all such $t$,

$$
\|u(\cdot, t(\cdot))\|_{L^{2}\left(\mathbb{D}^{n}\right)} \leq C\|f\|_{H^{s}} .
$$

In practice one looks at integral operators of the form

$$
R_{k} f(x)=\int e^{i\left(x \cdot y+t(x)|y|^{2}\right)} \theta_{k}(y) f(y) d y \quad k=1,2, \ldots
$$

where $\left\{\theta_{k}\right\}_{0}^{\infty}$ is a partition of unity such that $\operatorname{supp}\left(\theta_{k}\right) \subset\left\{y: 2^{k-1} \leq|y| \leq\right.$ $\left.2^{k+1}\right\}$ when $k \geq 1$. 
Proposition.$^{1}$ Suppose there is a $C$ and $s_{0} \geq 0$ such that

$$
\left\|R_{k} f\right\|_{L^{2}\left(\mathbb{D}^{n}\right)} \leq C 2^{s_{0} k} .
$$

Then for any $s>s_{0}$ there is $a C_{s}$, depending on $C$ and $s$, such that

$$
\|u(\cdot, t(\cdot))\|_{L^{2}\left(\mathbb{D}^{n}\right)} \leq C_{s}\|f\|_{H^{s}} .
$$

Thus we have reduced to the case of finding $L^{2}$ to $L^{2}$ estimates on a family of linear operators. This is a common task in harmonic analysis, and this particular one is aided by the similarites between the $R_{k}$ 's and a general class of operators, $\mathfrak{T}_{\lambda}: L^{2}\left(\mathbb{R}^{n}\right) \rightarrow L^{2}\left(\mathbb{R}^{n}\right)$, of the form

$$
\mathfrak{T}_{\lambda} f(x)=\int_{\mathbb{R}^{n}} e^{i \lambda \phi(x, y)} a(x, y) f(y) d y .
$$

Such operators, called oscillatory integral operators, are usually studied when the amplitude $a \in C_{0}^{\infty}\left(\mathbb{R}^{n} \times \mathbb{R}^{n}\right)$ and the phase function $\phi \in C^{\infty}\left(\mathbb{R}^{n} \times\right.$ $\mathbb{R}^{n}$ ), and one is concerned with the behavior of $\left\|\mathfrak{T}_{\lambda}\right\|$ as $\lambda \rightarrow \infty$.

There are two major differences, though, between $\mathfrak{T}_{\lambda}$ and $R_{k}$ that must be considered. Firstly, since the phase function in $R_{k}$ is not homogeneous, we cannot do a change of variables $y \rightarrow 2^{k} y$ to get into the form of $\mathfrak{T}_{\lambda}$. However, for the purpose of obtaining an $\epsilon$-improvement in pointwise convergence results when $n=2$, it is pointed out in $[\mathbf{B}]$ that it is sufficient to consider operators of the form

$$
T_{\lambda} f(x)=\int_{\mathbb{R}^{n}} \exp \left(i \lambda \frac{|x-y|^{2}}{t(x)-\bar{t}(y)}\right) a(x, y) f(y) d y,
$$

where $a \in C_{0}^{\infty}$ and $t$ and $\bar{t}$ are measurable functions such that $1 \leq \mid t(x)-$ $\bar{t}(y) \mid \leq 2$, and show that there exists an $\epsilon>0$ such that

$$
\left\|T_{\lambda} f\right\|_{2} \leq C \lambda^{-\epsilon}\|f\|_{2}, \quad C \text { independent of } t \text { and } \bar{t} .
$$

Such a result then implies an inequality as in (3) with $s_{0}<1 / 2$.

The second and more important difference is that the phase function in $R_{k}$ (and $T_{\lambda}$ ) is not smooth. The main results about $\mathfrak{T}_{\lambda}$ in $[\mathbf{G S}],[\mathbf{P S}]$ and [H1] involve only those cases when $\phi$ is smooth. Nevertheless (5) is plausible due to the following theorem, whose proof is based on ideas in $[\mathbf{B}]$.

Theorem 1. If $T_{\lambda}$ is as above then

$$
\left\|T_{\lambda} f\right\|_{L^{2}\left(\mathbb{R}^{n}\right)} \leq C \lambda^{-\frac{n-2}{4}}\|f\|_{L^{2}\left(\mathbb{R}^{n}\right)}
$$

\footnotetext{
${ }^{1}$ See $[\mathbf{K}]$ or $[\mathbf{B}]$.
} 
where $C$ is uniform over all measurable $t$ and $\bar{t}$ such that $1 \leq|t(x)-\bar{t}(y)| \leq 2$.

Of course when $n=2$ the estimate in (6) is trivial. The heart of [B] lies in dealing with the non-smoothness in the phase function of $T_{\lambda}$ to get the estimate in (5), which is an $\epsilon$ improvement of (6).

In this paper we discuss operators of the form $T_{\lambda}$ when the functions $t$ and $\bar{t}$ are assumed to be smooth. We begin by considering a special case of $T_{\lambda}$ when $\bar{t} \equiv 0$.

Theorem 2. Let $T_{\lambda}$ be as in (4) where $t$ is a smooth function such that $t \neq 0$, and $\bar{t} \equiv 0$.

(i) If $\frac{\nabla t(x)}{t(x)} \cdot(x-y)-1 \neq 0$ on $\operatorname{supp}(a)$, then $\left\|T_{\lambda} f\right\|_{2} \lesssim \lambda^{-n / 2}\|f\|_{2}$. Moreover the exponent of $\lambda$ is sharp.

(ii) In general, $\left\|T_{\lambda} f\right\|_{2} \lesssim \lambda^{-n / 2+1 / 4}\|f\|_{2}$.

(iii) For a given amplitude function $a \neq \equiv$, there are functions $t \in C^{\infty}$ such that the exponent of $\lambda$ in II is sharp.

This result follows from a more general theorem in $[\mathbf{G S}] .^{2}$ The proof given in this paper uses a similar approach to that in [GS], but our execution is different, and this difference allows us to consider operators, which are not oscillatory integral operators, like $R_{k}$, in another paper. And although Theorem 2 is a special case, an analogous theorem in its statement and proof is given, which is then used to prove Theorem 3 - the main result of this paper.

Theorem 3. Let $T_{\lambda}$ be as in (4) where $t$ and $\bar{t}$ are smooth functions such that $0<|t(x)-\bar{t}(y)|$. Then

(i) $\left\|T_{\lambda} f\right\|_{2} \lesssim \lambda^{-n / 2+1 / 2}$.

(ii) For a given amplitude function $a \not \equiv 0$, there are $t$ and $\bar{t}$ such that the bound in I is sharp.

\section{Preliminaries.}

Before proving our main theorems, we catalogue a number of important lemmas used in their proofs. These lemmas are variations of standard material, so their proofs are omitted and can be found in $[\mathbf{K}]$.

The following notation is used throughout.

$x, y, z$ and $\xi$ will denote variables in $\mathbb{R}^{n}$.

$x \cdot y$ is the inner product in $\mathbb{R}^{n}: x \cdot y=\sum_{1}^{n} x_{i} y_{i}$.

\footnotetext{
${ }^{2}$ See also $[\mathbf{K}]$ for details.
} 
$M^{t}$ denotes the transpose of the matrix $M$.

$\mathrm{H} f$ will denote the Hessian of $f$.

$\widehat{f}(\xi)=\int e^{-i x \cdot \xi} a(x) d x$ is the Fourier transform of $f$.

$\check{f}(\xi)=(2 \pi)^{-n} \int e^{i x \cdot \xi} f(x) d x$ is the inverse Fourier transform of $f$.

$\partial_{j}$ is the differential operator $\partial / \partial x_{j}$.

$\mathfrak{S}\left(\mathbb{R}^{n}\right)$ is the Schwartz class of functions on $\mathbb{R}^{n}$.

$B_{r}(p)=\left\{x \in \mathbb{R}^{n}:|x-p|<r\right\}$.

$\mathbb{D}^{n}$ denotes the unit ball in $\mathbb{R}^{n}$.

If $E \subset \mathbb{R}^{n}$ is measurable, then $|E|$ denotes the Lebesgue measure of $E$.

If $a(x, y)$ is a function of $x \in \mathbb{R}^{n}$ and $y \in \mathbb{R}^{m}$, then denote by $\operatorname{supp}_{y}(a)$ the projection onto the $y$-coordinates of the support of $a$. Let $\nabla_{y} a(x, y)$ denote the gradient of $a$ as a function of $y$ with $x$ held fixed. Similarly $\Delta_{y} a(x, y)=\sum \partial^{2} / \partial y_{j}^{2} a(x, y)$.

The expression $x \lesssim y$ will mean that there is a constant $C$, which does not depend on quantities that are otherwise to be kept track of, such that $x \leq C y$. Dependence on such quantities will be explicitly noted.

Given $x, y \in \mathbb{R}^{n}$, write $x=\left(x^{\prime}, x_{n}\right)$ and $y=\left(y^{\prime}, y_{n}\right)$, where $x^{\prime}$ and $y^{\prime}$ are in $\mathbb{R}^{n-1}$. Let $K(x, y)$ be a given bounded measurable function, which for our purposes will be assumed to have compact support, and define an operator $T: L^{2}\left(\mathbb{R}^{n}\right) \rightarrow L^{2}\left(\mathbb{R}^{n}\right)$ by

$$
T f(x)=\int_{\mathbb{R}^{n}} K(x, y) f(y) d y,
$$

whose adjoint is

$$
T^{*} f(y)=\int_{\mathbb{R}^{n}} \overline{K(z, y)} f(z) d z
$$

If we fix $x_{n}$ and $y_{n}$ and let $K_{x_{n} y_{n}}\left(x^{\prime}, y^{\prime}\right)=K\left(x^{\prime}, x_{n}, y^{\prime}, y_{n}\right)$, then we get a family of frozen operators, $T_{x_{n} y_{n}}: L^{2}\left(\mathbb{R}^{n-1}\right) \rightarrow L^{2}\left(\mathbb{R}^{n-1}\right)$ defined by

$$
T_{x_{n} y_{n}} f\left(x^{\prime}\right)=\int_{\mathbb{R}^{n-1}} K_{x_{n} y_{n}}\left(x^{\prime}, y^{\prime}\right) f\left(y^{\prime}\right) d y^{\prime} .
$$

Lemma 1.1. Suppose there exists a measurable function $\eta\left(x_{n}, y_{n}\right)$ such that for all $g \in L^{p}\left(\mathbb{R}^{n-1}\right)$ and for all $h \in L^{p}(\mathbb{R})$

$$
\begin{aligned}
& \left\|T_{x_{n} y_{n}} g\right\|_{L^{q}\left(\mathbb{R}^{n-1}\right)} \leq \eta\left(x_{n}, y_{n}\right)\|g\|_{L^{p}\left(\mathbb{R}^{n-1}\right)} \\
& \left\|\int \eta\left(x_{n}, y_{n}\right) h\left(y_{n}\right) d y_{n}\right\|_{L^{q}(\mathbb{R})} \leq C\|h\|_{L^{p}(\mathbb{R})} .
\end{aligned}
$$


Then for all $f \in L^{p}\left(\mathbb{R}^{n}\right)$

$$
\|T f\|_{L^{q}\left(\mathbb{R}^{n}\right)} \leq C\|f\|_{L^{p}\left(\mathbb{R}^{n}\right)} .
$$

Now consider

$$
T T^{*} f(x)=\int f(z)\left(\int K(x, y) \overline{K(z, y)} d y\right) d z .
$$

If $T_{x_{n}}: L^{2}\left(\mathbb{R}^{n}\right) \rightarrow L^{2}\left(\mathbb{R}^{n-1}\right)$ is the operator

$$
T_{x_{n}} f\left(x^{\prime}\right)=\int_{\mathbb{R}^{n}} K\left(x^{\prime}, x_{n}, y\right) f(y) d y,
$$

and $T_{z_{n}}^{*}: L^{2}\left(\mathbb{R}^{n-1}\right) \rightarrow L^{2}\left(\mathbb{R}^{n}\right)$

$$
T_{z_{n}}^{*} f(y)=\int_{\mathbb{R}^{n-1}} \overline{K\left(z^{\prime}, z_{n}, y\right)} f\left(z^{\prime}\right) d z^{\prime}
$$

is its adjoint, then clearly

$$
\begin{aligned}
\left(T T^{*}\right)_{x_{n} z_{n}} & =T_{x_{n}} T_{z_{n}}^{*}, \\
\left\|T_{x_{n}}\left(T_{z_{n}}\right)^{*} f\right\|_{L^{2}\left(\mathbb{R}^{n-1}\right)} & \leq\left\|T_{x_{n}}\right\|\left\|T_{z_{n}}^{*}\right\|\|f\|_{2} .
\end{aligned}
$$

Lemma 1.2.

$$
\begin{aligned}
\left\|T_{z_{n}}^{*} f\right\|_{L^{2}\left(\mathbb{R}^{n}\right)} & \leq\|f\|_{L^{2}\left(\mathbb{R}^{n-1}\right)}\left(\int_{-\infty}^{\infty}\left\|\left(T^{*}\right)_{z_{n} y_{n}}\right\|^{2} d y_{n}\right)^{1 / 2} . \\
\left\|T_{x_{n}} f\right\|_{L^{2}\left(\mathbb{R}^{n-1}\right)} & \leq\|f\|_{L^{2}\left(\mathbb{R}^{n}\right)}\left(\int_{-\infty}^{\infty}\left\|T_{x_{n} y_{n}}\right\|^{2} d y_{n}\right)^{1 / 2} .
\end{aligned}
$$

The method of stationary phase is of crucial importance to our endeavors.

Theorem 1.3. Suppose that $a \in \mathfrak{S}\left(\mathbb{R}^{n}\right)$. Then for any positive integer $k$,

$$
\begin{aligned}
\int_{\mathbb{R}^{n}} e^{i \lambda|y|^{2}} a(y) d y & \\
& =\left(\frac{i \lambda}{\pi}\right)^{-n / 2}\left(\sum_{j=0}^{k-1}(4 i \lambda)^{-j} \Delta^{j} a(0) / j !+\int_{\mathbb{R}^{n}} r_{k}\left(i|\xi|^{2} / 4 \lambda\right) \check{a}(\xi) d \xi\right),
\end{aligned}
$$

where $r_{k}(x)$ is the remainder of the $k$-th degree Taylor polynomial of $e^{x}$.

This well-known result is not usually expressed in this form. We find it convenient to include a form of the remainder term in the asymptotic expansion of the left hand side of (1.2) in powers of $\lambda$. See [H1], [St]. 
Remark 1.4. Note that $\left|r_{k}(x)\right| \leq|x|^{k} / k$ ! whenever $\operatorname{Re} x \leq 0$. Then an application of the Cauchy-Schwartz inequality shows that for any integer $s>n / 2$,

$$
\left|\int_{\mathbb{R}^{n}} r_{k}\left(i|\xi|^{2} / 4 \lambda\right) \check{a}(\xi) d \xi\right| \lesssim \lambda^{-k} \sum_{|\alpha| \leq 2 k+s}\left\|\mathrm{D}^{\alpha} a\right\|_{2}
$$

A corollary of Theorem 1.3 is needed to prove Theorem 3, which is a variable parameter version of Theorem 1.3.

Corollary 1.5. Suppose that $a$ is contained in a bounded subset $X$ of $\mathfrak{S}\left(\mathbb{R}^{n} \times \mathbb{R}^{m}\right)$. Then for any multi-index $\alpha$ and any $\lambda \geq 1$ there is a constant $C=C(\alpha, X)$ such that

$$
\sup _{z} \lambda^{n / 2}\left|\mathrm{D}_{z}^{\alpha} \int_{\mathbb{R}^{n}} e^{i \lambda|y|^{2}} a(y, z) d y\right| \leq C .
$$

We continue with a couple of lemmas about about $n \times n$ matrices.

Lemma 1.6. Let $M$ be an $n \times n$ matrix with entries $M_{i j}=\delta_{i}^{j}+a_{i} b_{j}$, where $\delta_{i}^{j}$ is the Kronecker delta and $a_{i}, b_{j} \in \mathbb{R}$. Then

(1) $\operatorname{det} M=1+\sum_{i=1}^{n} a_{i} b_{i}$,

(2) $\operatorname{rank}(M) \geq n-1$.

Lemma 1.7. Let $M$ be an $n \times n$ matrix of the form $M_{i j}=\delta_{i+1}^{j}+a_{i} b_{j}$. Then $\operatorname{det}(M)=a_{n} b_{1}$.

Finally we finish this section with a standard theorem about oscillatory integral operators

$$
\mathfrak{T}_{\lambda} f(x)=\int_{\mathbb{R}^{n}} e^{i \lambda \phi(x, y)} a(x, y) f(y) d y,
$$

where the amplitude $a \in C_{0}^{\infty}\left(\mathbb{R}^{n} \times \mathbb{R}^{n}\right)$, and the real-valued phase function $\phi \in C^{\infty}(X)$ with $X$ a neighborhood of $\operatorname{supp}(a)$. Just how rapidly $\left\|\mathfrak{T}_{\lambda}\right\|$ decays depends on the mixed Hessian of $\phi$, the $n \times n$ matrix $H_{\phi}(x, y)$ defined by

$$
\left(H_{\phi}(x, y)\right)_{i, j}=\frac{\partial^{2} \phi}{\partial x_{i} \partial y_{j}}(x, y) .
$$

The following is a sharpening of the result in [H2].

Theorem 1.8. Suppose that $H_{\phi}$ is non-singular on $\operatorname{supp}(a)$ and that the following quantities are uniformly bounded on $\operatorname{supp}(a)$ :

(i) $\left\|H_{\phi}^{-1}(x, y)\right\|$ 
(ii) $\left\|\nabla_{y} \mathrm{D}_{x}^{\alpha} \phi\right\|_{L^{\infty}(X)}$ for all $\alpha$ with $|\alpha|=2$

(iii) $\left\|\nabla_{x} \mathrm{D}_{y}^{\alpha} \phi\right\|_{L^{\infty}(X)}$ for all $\alpha$ with $|\alpha| \leq n+2$.

Then if $M=\max \left\{1,\left|\operatorname{supp}_{x}(a)\right|\right\}$ and

$$
M_{a}=\|a\|_{\infty}\left(M\left|\operatorname{supp}_{y}(a)\right|\left\{\sum_{|\alpha| \leq n+1} \sup _{x y z}\left|D_{y}^{\alpha} a(x, y) \overline{a(z, y)}\right|\right\}^{\frac{n}{n+1}}\right)^{1 / 2},
$$

then

$$
\left\|\mathfrak{T}_{\lambda} f\right\|_{2} \leq C M_{a} \lambda^{-n / 2}\|f\|_{2}
$$

where $C$ is bounded.

Remark 1.9. When bounding $\left\|H_{\phi}^{-1}\right\|$ uniformly from above it is convenient to use the classical theorem for the inverse of a matrix, $H_{\phi}^{-1}=$ $\left(\operatorname{det} H_{\phi}\right)^{-1}$ adj $H_{\phi}$. Then we need only bound $\operatorname{det} H_{\phi}$ uniformly from below and the entries of $H_{\phi}$ uniformly from above.

\section{Main Theorem: A Special Case.}

We let $a \in C_{0}^{\infty}\left(\mathbb{R}^{n} \times \mathbb{R}^{n}\right)$ be a fixed given function, and let $t \in C^{\infty}\left(\mathbb{R}^{n}\right)$ be such that $0 \neq t(x)$ on $\operatorname{supp}_{x}(a)$. We shall consider oscillatory integral operators $T_{\lambda}$ with phase function $\phi(x, y)=\frac{|x-y|^{2}}{t(x)}$ and catalogue the behavior of $\left\|T_{\lambda}\right\|$ below.

Remark 2.1. Since $\operatorname{supp}_{x}(a)$ is compact and $0 \neq t(x) \forall x \in \operatorname{supp}_{x}(a)$, there is a constant $c>0$ such that $c \leq|t(x)| \forall x \in \operatorname{supp}_{x}(a)$.

The best possible case is when $H_{\phi}$ is non-degenerate, and this situation is characterized in Theorem 2.2 below.

Theorem 2.2. Suppose $1-\frac{\nabla t(x)}{t(x)} \cdot(x-y) \neq 0$ on $\operatorname{supp}(a)$. Then for all $f$ $\left\|T_{\lambda} f\right\|_{2} \leq C \lambda^{-n / 2}\|f\|_{2}$, and the exponent of $\lambda$ is sharp. Moreover if we fix $c_{1}>0$, then $\exists c_{2}$ (which depends on $c_{1}$ and $\operatorname{supp}(a)$ ) such that the constant $C$ above is uniform over the set ${ }^{3}$

$$
\begin{aligned}
\Sigma & =\Sigma\left(c_{1}, c_{2}\right) \\
& =\left\{t \in C^{\infty}: c_{1} \leq|t(x)|,|\nabla t(x)| \leq c_{2}, \text { and }\|\mathrm{H} t(x)\| \leq c_{2} \forall x \in \operatorname{supp}(a)\right\} .
\end{aligned}
$$

\footnotetext{
${ }^{3}$ Here $\|\mathrm{H} t\|$ denotes the matrix norm of the $n \times n$ matrix $\mathrm{H}$.
} 
Proof. It is easy to calculate that

$$
\frac{\partial^{2} \phi}{\partial x_{i} \partial y_{j}}(x, y)=\frac{-2}{t(x)}\left(\delta_{i j}-\left(x_{j}-y_{j}\right) \frac{\partial_{i} t(x)}{t(x)}\right) .
$$

Then we see that $H_{\phi}$ is of the form described in Theorem 1.8, and we conclude that

$$
\operatorname{det} H_{\phi}(x, y)=\left(\frac{-2}{t(x)}\right)^{n}\left(1-\frac{\nabla t(x)}{t(x)} \cdot(x-y)\right) .
$$

The first part of the theorem follows now form Theorem 1.8; the second part will follow after a careful examination of the hypotheses in (i), (ii) and (iii) of Theorem 1.8 as they relate to $\phi$.

First note that if $c_{2}$ is chosen to be small enough, and $|\nabla t| \leq c_{2}$, then

$$
\left|\frac{\nabla t(x)}{t(x)} \cdot(x-y)\right| \leq c_{1}^{-1}|\nabla t(x)| 2 \operatorname{diam}(\operatorname{supp}(a)) \leq 1 / 2
$$

So if we assume that $t \in \Sigma$ for this choice of $c_{2}$ (and $c_{1}$ ), then $\left|\operatorname{det} H_{\phi}\right| \geq$ $c_{1}^{-n} 2^{n-1}$, and, given (2.1) and Remark1.9, $\left\|H_{\phi}^{-1}\right\|$ is uniformly bounded on $\operatorname{supp}(a)$ and over all $t \in \Sigma$. We claim also that $\left\|\nabla_{x} \mathrm{D}_{y}^{\alpha} \phi\right\|_{L^{\infty}}$ is uniformly bounded for all $\alpha$ with $|\alpha| \leq n+2$. In fact $|\alpha| \leq 2$ will suffice as all higher order derivatives vanish. The claim is evident from the form of $\phi$ as

$$
\frac{\partial}{\partial x_{j}} \mathrm{D}_{y}^{\alpha} \phi(x, y)=-\frac{\partial_{j} t(x)}{(t(x))^{2}} D_{y}^{\alpha}\left(|x-y|^{2}\right)
$$

and $t \in \Sigma$. Finally we check that $\left\|\nabla_{y} \mathrm{D}_{x}^{\alpha} \phi\right\|_{L^{\infty}}$ is also uniformly bounded when $\alpha=2$ since

$$
\frac{\partial^{3} \phi(x, y)}{\partial x_{i} \partial x_{j} \partial y_{k}}=\frac{-2}{t(x)^{2}}\left(\partial_{j} t(x) \delta_{i}^{k}-\delta_{j}^{k} \partial_{i} t(x)-\left(x_{k}-y_{k}\right)+\left(x_{k}-y_{k}\right) \frac{\partial_{i} t(x) \partial_{j} t(x)}{t(x)}\right) .
$$

The hypotheses of Theorem 1.8 being satisfied, the theorem is proven.

The more interesting case is when $\operatorname{det} H_{\phi}=0$. Lemma 1.6 readily gives the estimate $\left\|T_{\lambda} f\right\| \lesssim \lambda^{-n / 2+1 / 2}\|f\|_{2}$ in this case. But before proving a stronger estimate, a few remarks are in order.

Remark 2.3. If we cover $\operatorname{supp}_{x}(a)$ with balls of radius $\delta$ and take a partition of unity subordinate to these balls, we may assume that $\operatorname{diam}\left(\operatorname{supp}_{x}(a)\right)$ $<\delta$ without any loss of generality if we provide that $\delta$ does not depend on $\lambda$. Then $\delta$ is chosen to be as small as necessary to assist in technical matters. 
Remark 2.4. (2.2) says that we may assume (after a partition of unity) that on the support of $a, 1 \lesssim|\nabla t(x)|$. Otherwise $H_{\phi}$ is non-singular, and we may again appeal to Theorem 1.8.

Remark 2.5. If $A$ is a rotation then the change of variables $(x, y) \rightarrow$ $(A x, A y)$ "preserves" $\phi$ in the sense that $\phi(A x, A y)=\frac{|x-y|^{2}}{t \circ A}$ is of the same form as $\phi(x, y)$, the form of phase function presently under consideration, with $t$ replaced by $t \circ A$. Similarly if $A$ is a translation, $\phi$ is again "preserved" under the operation $\phi(x, y) \rightarrow \phi(A x, A y)$. Since such transformations are measure preserving, the norm of the oscillatory integral operator with phase function $\phi(x, y)$ and amplitude $a(x, y)$ is the same as the one with phase $\phi(A x, A y)$ and amplitude $a(A x, A y)$.

Theorem 2.6. Let $\phi$ be as above. Then in general

$$
\left\|T_{\lambda} f\right\|_{2} \leq C \lambda^{-n / 2+1 / 4}\|f\|_{2}
$$

Proof. Let $x \in \operatorname{supp}_{x}(a)$ be given. By Remark 2.4 we may find a rotation $A_{x}$ such that $\nabla t(x) A_{x}$ is parallel to the $n$-th unit vector $e_{n}$ in $\mathbb{R}^{n}$. Let $z=A_{x}^{-1} x$ and $t_{A_{x}}=t \circ A_{x}$. Then $\nabla t_{A_{x}}(z)$ is parallel to $e_{n}$, and so we may assume without loss of generality that $\nabla t_{A_{x}}(z)=e_{n}$. Furthermore it is clear that $\left|\nabla t_{A_{x}}\right| \gtrsim 1$ on $\operatorname{supp}(a)$. Then there is a neighborhood $U_{x}$ of $z$, a neighborhood $V_{x}$ of $x$ and a diffeomorphism $\rho_{x}: V_{x} \rightarrow U_{x}$ such that $t_{A_{x}} \circ \rho_{x}(w)=w_{n}$ for all $w \in V_{x}$. Moreover $\mathrm{D} \rho_{x}(z)=\mathrm{I}$, and we may assume that $\operatorname{diam}\left(U_{x}\right)$ and $\operatorname{diam}\left(V_{x}\right)$ are as small as necessary. Let $\widetilde{U}_{x}=A\left(U_{x}\right)$, and take a finite subcover $\left\{\widetilde{U}_{x_{i}}\right\}_{i=1}^{m}$ of $\operatorname{supp}_{x}(a)$. By Remark 2.3 we may assume that $\operatorname{supp}_{x}(a) \subset \widetilde{U}_{x_{1}}$ for example. Since $A_{x_{1}}$ is a rotation, by Remark 2.5 we may assume therefore that there is a ball $B_{\delta}\left(x_{0}\right)$ and a diffeomorphism $\rho: B_{\delta}\left(x_{0}\right) \rightarrow \operatorname{supp}_{x}(a)$ such that $t \circ \rho(x)=x_{n}$, and $\mathrm{D} \rho\left(x_{0}\right)=\mathrm{I}$.

Given this, it suffices after a change of variables to consider the operator

$$
\widetilde{T}_{\lambda} f(x)=\int_{\mathbb{R}^{n}} e^{i \lambda\left(\frac{|\rho(x)-y|^{2}}{x_{n}}\right)} a(\rho(x), y) f(y) d y
$$

If $S_{\lambda}=\widetilde{T}_{\lambda} \widetilde{T}_{\lambda}^{*}$, then $S_{\lambda}$ is an integral operator with kernel

$$
K_{\rho}(x, z)=\int_{\mathbb{R}^{n}} \exp \left(i \lambda\left\{\frac{|\rho(x)-y|^{2}}{x_{n}}-\frac{|\rho(z)-y|^{2}}{z_{n}}\right\}\right) a(\rho(x), y) \overline{a(\rho(z), y)} d y .
$$

We must show that

$$
\left\|S_{\lambda} f\right\|_{2} \lesssim \lambda^{-n+1 / 2}\|f\|_{2}
$$


Let $\psi \in C_{0}^{\infty}\left(B_{1}(0)\right)$ be such that $\psi \equiv 1$ on $B_{1 / 2}(0)$, and let $\widetilde{\psi}=1-\psi$. Then

$$
\begin{aligned}
S_{\lambda} f(x)= & \int_{\mathbb{R}^{n}} f(z) K_{\rho}(x, z) \psi\left(\frac{x_{n}-z_{n}}{\epsilon}\right) d z \\
& +\int_{\mathbb{R}^{n}} f(z) K_{\rho}(x, z) \widetilde{\psi}\left(\frac{x_{n}-z_{n}}{\epsilon}\right) d z \\
= & S_{\lambda}^{1} f(x)+S_{\lambda}^{2} f(x),
\end{aligned}
$$

where $\epsilon$ is to be chosen.

First consider $S_{\lambda}^{1}$ and its corresponding frozen operators

$$
\begin{aligned}
\left(S_{\lambda}^{1}\right)_{x_{n} z_{n}} f\left(x^{\prime}\right) & =\psi\left(\frac{x_{n}-z_{n}}{\epsilon}\right) \int_{\mathbb{R}^{n-1}} f\left(z^{\prime}\right)\left(K_{\rho}\right)_{x_{n} z_{n}}\left(x^{\prime}, z^{\prime}\right) d z^{\prime} \\
& =\psi\left(\frac{x_{n}-z_{n}}{\epsilon}\right)\left(\widetilde{T}_{\lambda} \widetilde{T}_{\lambda}^{*}\right)_{x_{n} z_{n}} f\left(x^{\prime}\right) .
\end{aligned}
$$

We consider $\left(\widetilde{T}_{\lambda}\right)_{x_{n} y_{n}}$ for fixed $x_{n}$ and $y_{n}$. The $(n-1) \times(n-1)$ matrix

$$
\left(\frac{\partial^{2}}{\partial x_{i} \partial y_{j}}|\rho(x)-y|^{2}\right)_{i, j=1}^{n-1}=\left(-2 \mathrm{D}_{i} \rho_{j}(x)\right)_{i, j=1}^{n-1}
$$

is the mixed Hessian for $\left(\widetilde{T}_{\lambda}\right)_{x_{n} y_{n}}$. As noted above, when $x=x_{0}$ this is -2 times the $(n-1) \times(n-1)$ identity matrix. So in a small neighborhood of $x_{0}$ the determinant of the above matrix does not vanish (see Remark 2.3). Hence

$$
\left\|\left(\widetilde{T}_{\lambda}\right)_{x_{n} y_{n}}\right\| \lesssim \lambda^{-(n-1) / 2} \chi\left(x_{n}, y_{n}\right)\|f\|_{2}
$$

where $\chi$ is compactly supported. Then this along with (1.1) and Lemma 1.2 implies that

$$
\left\|\left(S_{\lambda}^{1}\right)_{x_{n} y_{n}} f\right\|_{L^{2}\left(\mathbb{R}^{n-1}\right)} \lesssim \lambda^{-n+1} \chi^{\prime}\left(x_{n}, z_{n}\right) \psi\left(\frac{x_{n}-z_{n}}{\epsilon}\right)\|f\|_{L^{2}\left(\mathbb{R}^{n-1}\right)},
$$

where $\chi^{\prime}$ is also compactly supported. By Schur's Lemma and Lemma 1.1 we have

$$
\left\|S_{\lambda}^{1} f\right\|_{2} \lesssim \lambda^{-n+1} \epsilon\|f\|_{2} .
$$

Now consider $S_{\lambda}^{2}$ and its corresponding frozen operators $\left(S_{\lambda}^{2}\right)_{x_{n} z_{n}}$ for fixed $x_{n}$ and $z_{n}$. Note that

$$
\begin{aligned}
\frac{|\rho(x)-y|^{2}}{x_{n}}- & \frac{|\rho(z)-y|^{2}}{z_{n}} \\
& =\left(\frac{1}{x_{n}}-\frac{1}{z_{n}}\right)|y-F(x, z)|^{2}-\frac{\left|\rho\left(x^{\prime}, x_{n}\right)-\rho\left(z^{\prime}, z_{n}\right)\right|^{2}}{x_{n}-z_{n}}
\end{aligned}
$$


where

$$
F(x, z)=\frac{z_{n} \rho(x)-x_{n} \rho(z)}{x_{n}-z_{n}}
$$

Let

$$
\begin{aligned}
A(x, z, y) & =a(\rho(x), y) \overline{a(\rho(z), y)}, \\
\mu & =\lambda\left(\frac{1}{x_{n}}-\frac{1}{z_{n}}\right) .
\end{aligned}
$$

Then

$$
\begin{aligned}
K_{\rho}\left(x^{\prime}, z^{\prime}\right)_{x_{n} z_{n}}=\widetilde{\psi} & \left(\frac{x_{n}-z_{n}}{\epsilon}\right) \exp \left(i \frac{\lambda}{z_{n}-x_{n}}|\rho(x)-\rho(z)|^{2}\right) \\
& \cdot \int_{\mathbb{R}^{n}} e^{i \mu|y|^{2}} A(x, z, y+F(x, z)) d y
\end{aligned}
$$

is the kernel of $\left(S_{\lambda}^{2}\right)_{x_{n} z_{n}}$. By Theorem 1.3, for fixed $N$ to be chosen,

$$
\begin{aligned}
& \int_{\mathbb{R}^{n}} e^{i \mu|y|^{2}} A(x, z, y+F(x, z)) d y \\
& =\left(\frac{i \mu}{\pi}\right)^{-n / 2}\left(\sum_{j=0}^{N-1}(4 i \mu)^{-j} \Delta_{y}^{j} A(x, z, F(x, z)) / j !\right. \\
& \left.\quad+\int_{\mathbb{R}^{n}} r_{N}\left(i|\xi|^{2} / 4 \mu\right) e^{-i \xi \cdot F(x, z)} \check{A}(x, z, \xi) d \xi\right),
\end{aligned}
$$

where $\check{A}$ denotes the inverse Fourier transform in the last variable. In view of (2.10) and (2.11), $\left(S_{\lambda}^{2}\right)_{x_{n} z_{n}}$ is a sum of oscillatory integral operators

$$
\left(\frac{i}{\pi}\right)^{-n / 2} \widetilde{\psi}\left(\frac{x_{n}-z_{n}}{\epsilon}\right) \sum_{j=0}^{N} \frac{\mu^{-n / 2-j}}{(4 i) j !} R_{\lambda^{\prime}}^{j}, \quad \lambda^{\prime}=\frac{\lambda}{x_{n}-z_{n}},
$$

where

$$
\begin{aligned}
R_{\lambda^{\prime}}^{j} f\left(x^{\prime}\right)= & \int_{\mathbb{R}^{n}} e^{i \lambda^{\prime}|\rho(x)-\rho(z)|^{2}}\left(\Delta_{y}^{j} A\right)(x, z, F(x, z)) f\left(z^{\prime}\right) d z^{\prime} \\
R_{\lambda^{\prime}}^{N} f\left(x^{\prime}\right)= & \int_{\mathbb{R}^{n}} e^{i \lambda^{\prime}|\rho(x)-\rho(z)|^{2}} \mu^{N} \\
& \cdot\left(\int_{\mathbb{R}^{n}} r_{N}\left(i|\xi|^{2} / 4 \mu\right) e^{-i \xi \cdot F(x, z)} \check{A}(x, z, \xi) d \xi\right) f\left(z^{\prime}\right) d z^{\prime} .
\end{aligned}
$$


Each $R_{\lambda^{\prime}}^{j}$ has phase function of the form $\left|\rho\left(x^{\prime}, x_{n}\right)-\rho\left(z^{\prime}, z_{n}\right)\right|^{2}$ for fixed $x_{n}$ and $z_{n}$. Look at the mixed Hessian:

$$
\left(\frac{\partial^{2}}{\partial x_{i} \partial z_{j}}\left|\rho\left(x^{\prime}, x_{n}\right)-\rho\left(z^{\prime}, z_{n}\right)\right|^{2}\right)_{i, j=1}^{n-1}=\left(-2 \sum_{k} \partial_{i} \rho_{k}(x) \partial_{j} \rho_{k}(z)\right)_{i, j=1}^{n-1} .
$$

Since $\mathrm{D} \rho\left(x_{0}\right)=\mathrm{I}$,

$$
\operatorname{det}\left(\frac{\partial^{2}}{\partial x_{i} \partial z_{j}}\left|\rho\left(x^{\prime}, x_{n}\right)-\rho\left(z^{\prime}, z_{n}\right)\right|^{2}\right)_{i, j=1}^{n-1}=(-2)^{n-1} \quad \text { when } x=z=x_{0} .
$$

So we may assume that this mixed Hessian is non-degenerate (see Remark 2.3). Now for $j=1, \ldots, N-1, R_{\lambda^{\prime}}^{j}$ has amplitude $\Delta_{y}^{j} A(x, z, F(x, z))$. Since

$$
\left|\mathrm{D}_{z^{\prime}}^{\alpha} F(x, z)\right| \lesssim\left|x_{n}-z_{n}\right|^{-1}
$$

we see that

$$
\sup _{x^{\prime} z^{\prime}}\left|\mathrm{D}_{z^{\prime}}^{\alpha}\left(\Delta_{y}^{j} A(x, z, F(x, z)) \overline{\Delta_{y}^{j} A}(w, z, F(w, z))\right)\right| \lesssim\left|x_{n}-z_{n}\right|^{-|\alpha|} .
$$

To apply Theorem 1.8 we must calculate, for fixed $x_{n}$ and $z_{n}$, the volume of $\operatorname{supp}_{z^{\prime}}\left(\Delta_{y}^{j} A(x, z, F(x, z))\right)$. Note that by the properties of $\operatorname{supp}(A)$ we must have that $|F(x, z)| \lesssim 1-$ i.e., $\left|x_{n} \rho(z)-z_{n} \rho(x)\right| \lesssim\left|x_{n}-z_{n}\right|$. This says that for fixed $x, \rho(z)$ is in the ball of radius $\frac{1}{x_{n}}\left|x_{n}-z_{n}\right|$ centered at $\frac{z_{n}}{x_{n}} \rho(x)$. Since $\left|x_{n}\right|$ is bounded from below (see Remark 2.1) and $\rho$ is a diffeomorphism, $z$ lies in a set of diameter $\sim\left|x_{n}-z_{n}\right|$. So

$$
\left|\operatorname{supp}_{z^{\prime}} \Delta_{y}^{j} A(x, z, F(x, z))\right| \sim\left|x_{n}-z_{n}\right|^{n-1} \quad \text { for } j=0, \ldots, N-1 .
$$

Putting (2.15) and (2.16) into (1.4) (recalling that $\left.\lambda^{\prime}=\frac{\lambda}{\left(x_{n}-z_{n}\right)}\right)$ gives that for $j=1, \ldots, N-1$,

$$
\left\|R_{\lambda^{\prime}}^{j} f\right\|_{L^{2}\left(\mathbb{R}^{n-1}\right)} \lesssim \lambda^{\frac{-(n-1)}{2}}\left|x_{n}-z_{n}\right|^{\frac{(n-1)}{2}} \chi\left(x_{n}, z_{n}\right)\|f\|_{L^{2}\left(\mathbb{R}^{n-1}\right)},
$$

where $\chi$ has compact support.

Coming to $R_{\lambda^{\prime}}^{N}$, it has amplitude, call it $A_{N}(x, z)$, equal to

$$
\mu^{N} \int_{\mathbb{R}^{n}} r_{N}\left(i|\xi|^{2} / 4 \mu\right) e^{-i \xi \cdot F(x, z)} \check{A}(x, z, \xi) d \xi .
$$


This means that in view of the Remark 1.4 and (2.14)

$$
\sup _{z^{\prime}}\left|\mathrm{D}_{z^{\prime}}^{\alpha} A_{N}(x, z) \overline{A_{N}(w, z)}\right| \lesssim\left|x_{n}-z_{n}\right|^{-|\alpha|} .
$$

Then (2.18) implies that

$$
\left\|R_{\lambda^{\prime}}^{N} f\right\|_{L^{2}\left(\mathbb{R}^{n-1}\right)} \lesssim \lambda^{\frac{-(n-1)}{2}} \chi\left(x_{n}, z_{n}\right)\|f\|_{L^{2}\left(\mathbb{R}^{n-1}\right)} .
$$

Looking at (2.12), (2.17) and (2.19) we see that

$$
\left\|\left(S_{\lambda}^{2}\right)_{x_{n} z_{n}} f\right\|_{L^{2}\left(\mathbb{R}^{n-1}\right)} \lesssim \widetilde{\psi}\left(\frac{x_{n}-z_{n}}{\epsilon}\right) \chi\left(x_{n}, z_{n}\right)
$$

where $\chi$ is compactly supported. Now we apply Lemma 1.1 to obtain

$$
\begin{aligned}
\left\|S_{\lambda}^{2} f\right\|_{L^{2}\left(\mathbb{R}^{n}\right)} \lesssim & \|f\|_{L^{2}\left(\mathbb{R}^{n}\right)} \\
& \cdot\left(\lambda^{-n+1 / 2}\right. \\
& +\sum_{j=1}^{N-1} \lambda^{-n+1 / 2-j} \epsilon^{1 / 2-j} \\
& \left.+\lambda^{-n+1 / 2-N} \epsilon^{-n / 2-N+1}\right) .
\end{aligned}
$$

In consideration of $(2.6)$ and $\left(2.21^{\prime \prime}\right)$ set

$$
\epsilon=\lambda^{-\frac{2 N+1}{n+2 N}} .
$$

In this case

$$
\lambda^{-n+1} \epsilon=\lambda^{-n+1 / 2-N} \epsilon^{-n / 2-N+1}=\lambda^{-n+1 / 2} \lambda^{\frac{n-2(N+2)}{2(n+2 N)}} .
$$

Also, it is easy to check, in consideration of $\left(2.21^{\prime}\right)$, that

$$
\lambda^{-n / 2+1 / 2-j} \epsilon^{1 / 2-j} \leq \lambda^{-n+1 / 2}
$$

for this choice of $\epsilon$. Evidently (2.21) is the main term in the estimate of $\left\|S_{\lambda}\right\|$ provided that $n \leq 2(N+2)$. We choose $N$ as so and then let $\epsilon$ be as 
in (2.22) for this choice of $N$. Now (2.3) is demonstrated, and the proof of Theorem 2.6 is complete.

Given the general nature of Theorem 2.6, it is natural to ask whether or not the result is sharp. We shall find in the next theorem that we may not always be able to improve the exponent of $\lambda$ in Theorem 2.6.

Theorem 2.7. For a given amplitude function $a \not \equiv 0$, there are $t \in C^{\infty}$ such that the exponent of $\lambda$ in Theorem 2.6 is sharp.

Proof. By assumption there is a point $\left(x_{0}, y_{0}\right)$ such that $a\left(x_{0}, y_{0}\right) \neq 0$. After perhaps a translation and a rotation - in view of Remark 2.5 - we may assume that $x_{0}=e_{n}$, the $n$-th unit vector in $\mathbb{R}^{n}$, and $y_{0}=0$. Then let $t(x)=$ $x_{n}$, and note that we may assume $x_{n} \neq 0$ on $\operatorname{supp}(a)$. Let $f_{\epsilon}(y)=f\left(y^{\prime}\right) \tilde{f}\left(y_{n}\right)$, where $0 \leq f \in C_{0}^{\infty}\left(\mathbb{R}^{n-1}\right), f \equiv 1$ on $\operatorname{supp}(a)$ and $\tilde{f}\left(y_{n}\right)=e^{i 2 \lambda y_{n}} \chi_{[-\epsilon, \epsilon]}\left(y_{n}\right)$. Then

$$
\begin{aligned}
e^{-i \lambda x_{n}} T_{\lambda} f(x)= & \int_{\mathbb{R}^{n-1}} e^{\frac{\lambda}{x_{n}}\left|x^{\prime}-y^{\prime}\right|^{2}} f\left(y^{\prime}\right) \int_{-\epsilon}^{\epsilon} e^{i \lambda \frac{y_{n}^{2}}{x_{n}}} a\left(x, y^{\prime}, y_{n}\right) d y_{n} d y^{\prime} \\
= & \left(\frac{i \lambda}{x_{n} \pi}\right)^{-(n-1) / 2} 2 \epsilon f\left(x^{\prime}\right) \frac{1}{2 \epsilon} \int_{-\epsilon}^{\epsilon} a\left(x, x^{\prime}, y_{n}\right) d y_{n} \\
& +\left(\frac{i \lambda}{x_{n} \pi}\right)^{-(n-1) / 2} f\left(x^{\prime}\right) \int_{-\epsilon}^{\epsilon}\left(e^{i \lambda \frac{y_{n}^{2}}{x_{n}}}-1\right) a\left(x, x^{\prime}, y_{n}\right) d y_{n} \\
& +\left(\frac{i \lambda}{x_{n} \pi}\right)^{-(n-1) / 2} 2 \epsilon \int_{\mathbb{R}^{n-1}} r_{1}\left(i x_{n}\left|\xi^{\prime}\right|^{2} / 4 \lambda\right) \\
& \cdot\left(f\left(x^{\prime}+\cdot\right) \frac{1}{2 \epsilon} \int_{-\epsilon}^{\epsilon} e^{i \lambda \frac{y_{n}^{2}}{x_{n}}} a\left(x, \cdot+x^{\prime}, y_{n}\right) d y_{n}\right) \frown\left(\xi^{\prime}\right) d \xi^{\prime} \\
= & \mathrm{I}+\mathrm{II}+\mathrm{III} .
\end{aligned}
$$

Now it is easily seen that

$$
|\mathrm{II}| \lesssim \lambda^{-n / 2+3 / 2} \epsilon^{3}
$$

and by (1.3)

$$
\begin{aligned}
|\mathrm{III}| & \lesssim \epsilon \lambda^{-n / 2-1 / 2} \sum_{|\alpha| \leq 2+n / 2}\left\|\mathrm{D}_{y^{\prime}}^{\alpha} f\left(x^{\prime}+\cdot\right) \frac{1}{2 \epsilon} \int_{-\epsilon}^{\epsilon} e^{i \lambda \frac{y_{n}^{2}}{x_{n}}} a\left(x, \cdot, y_{n}\right) d y_{n}\right\|_{L^{2}\left(\mathbb{R}^{n-1}\right)} \\
& \lesssim \epsilon \lambda^{-n / 2-1 / 2} .
\end{aligned}
$$

Supposing that $\epsilon$ is small we have that

$$
|\mathrm{I}| \gtrsim \epsilon \lambda^{-n / 2+1 / 2}\left|\frac{1}{2 \epsilon} \int_{-\epsilon}^{\epsilon} a\left(x, x^{\prime}, y_{n}\right) d y_{n}\right| \gtrsim \epsilon \lambda^{-n / 2+1 / 2}
$$


on a set of positive measure in $x$-space. If we let $\epsilon=c \lambda^{-1 / 2}$ where $\mathrm{c}$ is a small constant independent of $\lambda$ (i.e. let $\epsilon \lambda^{-n / 2+1 / 2}=\epsilon^{3} \lambda^{-n / 2+3 / 2}$ ), then clearly

$$
\left\|T_{\lambda}^{\prime} f_{\epsilon}\right\|_{2} \gtrsim \lambda^{-n / 2} \quad \text { and } \quad\left\|f_{\epsilon}\right\|_{2}=\lambda^{-1 / 4} \text {. }
$$

So

$$
\frac{\left\|T_{\lambda}^{\prime} f_{\epsilon}\right\|_{2}}{\left\|f_{\epsilon}\right\|_{2}} \gtrsim \lambda^{-n / 2+1 / 4}
$$

as desired.

Remark 2.8. Using a result of Pan and Sogge $[\mathbf{P S}]$ it is a routine matter to show that if $H t(x)(x-y) \cdot(x-y) \neq 0$ on $\operatorname{supp}(a)$ where $H t(x)$ denotes the Hessian of $t$ at $x$, then $\left\|T_{\lambda}^{\prime} f\right\|_{2} \leq C \lambda^{-n / 2+1 / 6}\|f\|_{2}$.

To conclude this section we consider a variant of the operator previously considered. We let $a \in C_{0}^{\infty}\left(\mathbb{R}^{n} \times \mathbb{R}^{n}\right)$ be a given fixed amplitude function, but we let $t$ denote any smooth function. Now we shall study the oscillatory integral operator $T_{\lambda}^{\prime}$, with phase function $\phi(x, y)=-2 x \cdot y+t(x)|y|^{2}$. Its purpose is to provide a means of understanding $T_{\lambda}$ in its full generality. The phase function involved is more suited to this task from a technical standpoint. We could have just as easily applied Theorem 2.6 to the more general case of $T_{\lambda}$, but the proofs of Theorems 2.9, 2.10 and 2.11 follow closely those of their counterparts, Theorems 2.2, 2.6 and 2.7, and we get these results almost for free. The proofs are therefore omitted and can be found in $[\mathbf{K}]$.

Theorem 2.9. If $1-\nabla t(x) \cdot y \neq 0$ on $\operatorname{supp}(a)$ then $\left\|T_{\lambda}^{\prime} f\right\|_{2} \leq C \lambda^{-n / 2}\|f\|_{2}$, and the exponent of $\lambda$ is sharp. Moreover $\exists c$ such that the constant $C$ is uniform over the set

$$
\Sigma=\Sigma(c)=\left\{t \in C^{\infty}:|\nabla t(x)|,\|\mathrm{H} t\| \leq c \forall x \in \operatorname{supp}(a)\right\}
$$

Theorem 2.10. Let $\phi$ be as above. Then in general

$$
\left\|T_{\lambda}^{\prime} f\right\|_{2} \leq C \lambda^{-n / 2+1 / 4}\|f\|_{2} .
$$

Theorem 2.11. For a given amplitude function $a \not \equiv 0$, there are $t \in C^{\infty}$ such that the exponent of $\lambda$ in Theorem 2.10 is sharp. 


\section{Main Theorem.}

We now turn our attention to the family of operators $T_{\lambda}$ with phase function of the form $\phi(x, y)=\frac{|x-y|^{2}}{t(x)-\bar{t}(y)}$ where $t$ and $\bar{t}$ are smooth functions such that $0<|t(x)-\bar{t}(y)|$. In what follows we will always take $a$ to be a fixed amplitude function with compact, connected support. Hence we may assume without loss of generality that there is a constant $c>0$ such that $c \leq t(x)-\bar{t}(y)$ on $\operatorname{supp}(a)$. This will be our general setup through this section.

We shall find it to be possible that rank $H_{\phi}=n-2$, and therefore the ideas in Section 2 do not carry over into the analysis of $\left\|T_{\lambda}\right\|$. Instead, we would like to consider $T_{\lambda}$ as a composition of operators whose factors are known to us, these factors already having been studied in the previous section. We may then use the estimate in Theorem 2.10 to get sharp results for $T_{\lambda}$. In actual fact, though, we will not realize $T_{\lambda}$ directly as a composition, but nearly so. The composition of operators we consider has the same phase function as $T_{\lambda}$ but has a different amplitude. The transition to $T_{\lambda}$ in Theorem 3.3 from Theorem 2.10 is facilitated by Lemma 3.2, which allows us to compare oscillatory integral operators with the same phase function but different amplitudes.

We begin with the following result about the singularities of $H_{\phi}$.

Proposition 3.1. Let $\phi$ be as above. Then $\operatorname{rank} H_{\phi}(x, y) \geq n-2$, and moreover we have that $\operatorname{rank} H_{\phi}(x, y)=n-2$ if and only if

(i) $1-\frac{\nabla t(x) \cdot(x-y)}{t(x)-\bar{t}(y)}=0$.

(ii) $1-\frac{\nabla \bar{t}(y) \cdot(x-y)}{t(x)-\bar{t}(y)}=0$.

(iii) $\nabla t(x) \cdot \nabla \bar{t}(y)=0$.

Proof. We begin by noting that

$$
\begin{aligned}
& \frac{\partial^{2} \phi(x, y)}{\partial x_{i} \partial y_{j}}=\frac{-2}{t(x)-\bar{t}(y)}\left(\delta_{i}^{j}-\frac{1}{t(x)-\bar{t}(y)}\right. \\
& \left.(3.1) \quad \cdot\left(\frac{\partial}{\partial y_{j}} \bar{t}(y)\left(x_{i}-y_{i}\right)+\frac{\partial}{\partial x_{i}} t(y)\left(x_{j}-y_{j}\right)-|x-y|^{2} \frac{\partial_{i} t(x) \partial_{j} \bar{t}(y)}{t(x)-\bar{t}(y)}\right)\right) .
\end{aligned}
$$

Suppose first that $\nabla \bar{t}(y)=0$. Then from (3.1),

$$
\left(H_{\phi}(x, y)\right)_{i j}=\frac{-2}{t(x)-\bar{t}(y)}\left(\delta_{i}^{j}-\frac{\left(x_{j}-y_{j}\right)}{t(x)-\bar{t}(y)} \partial_{i} t(x)\right),
$$

and such a matrix has rank $\geq n-1$ by Lemma 1.6. So we may assume that $\nabla \bar{t}(y) \neq 0$. In fact we may assume that $\nabla \bar{t}(y) \| e_{n}$. For let $A$ be a rotation of 
$\mathbb{R}^{n}$ such that $\nabla \bar{t}(y) A \| e_{n}$. Consider $\phi_{A}(z, w)=\phi(A z, A w)=\frac{|z-w|^{2}}{t_{A}(z)-\bar{t}_{A}(w)}$, $t_{A}=t \circ A, \bar{t}_{A}=\bar{t} \circ A$. Then $H_{\phi_{A}}=A^{t} H_{\phi}(A z, A w) A$. For given $x$ and $y$, let $z=A^{-1} x, w=A^{-1} y$. Then clearly $\operatorname{rank} H_{\phi}(x, y)=\operatorname{rank} H_{\phi_{A}}(z, w)$. Moreover, it is routine to check that

(i') $\quad 1-\frac{\nabla t(x) \cdot(x-y)}{t(x)-\bar{t}(y)}=1-\frac{\nabla t_{A}(z) \cdot(z-w)}{t_{A}(z)-\bar{t}_{A}(w)}$.

(ii') $1-\frac{\nabla \bar{t}(x) \cdot(x-y)}{t(x)-\bar{t}(y)}=1-\frac{\nabla \bar{t}_{A}(w) \cdot(z-w)}{t_{A}(z)-\bar{t}_{A}(w)}$.

(iii') $\nabla t(x) \cdot \nabla \bar{t}(y)=\nabla t_{A}(z) \cdot \nabla \bar{t}_{A}(w)$.

Since $\nabla \bar{t}(y) \| e_{n}$, we have that the $(n-1) \times(n-1)$ submatrix $H_{\phi}^{\prime}$ of $H_{\phi}$ formed by deleting the last row and last column is of the form

$\left(H_{\phi}^{\prime}(x, y)\right)_{i j}=\frac{-2}{t(x)-\bar{t}(y)}\left(\delta_{i}^{j}-\left(x_{j}-y_{j}\right) \partial_{j} t(x)\right) \quad 1 \leq i \leq n-1,1 \leq j \leq n-1$.

This submatrix has rank $\geq n-2$ (by Lemma 1.6); hence so does $H_{\phi}$.

Suppose that (i), (ii) and (iii) are satisfied. Since $\nabla \bar{t}(y) \| e_{n}$ and (iii) holds, we have that $\partial_{n} t(x)=0$. So by (ii),

$$
H_{\phi}(x, y)=\left(\begin{array}{ccc|c} 
& & & * \\
& H_{\phi}^{\prime}(x, y) & & \vdots \\
& & & * \\
\hline 0 & \cdots & 0 & 0
\end{array}\right) .
$$

Since (i) (or (ii)) holds, $x-y \neq 0$. Clearly,

$$
\left(0,0, \ldots, x_{n}-y_{n}\right) H_{\phi}(x, y)=0,
$$

while by (i)

$$
\left(x^{\prime}-y^{\prime}, 0\right) H_{\phi}(x, y)=0 .
$$

So rank $H_{\phi}(x, y)=n-2$.

Now suppose that $\operatorname{rank} H_{\phi}(x, y)=n-2$ (assuming again that $\left.\nabla \bar{t}(y) \neq 0\right)$. In particular, by Lemma 1.6, $H_{\phi}^{\prime}$ has rank $n-2$, and $\nabla_{x^{\prime}} t(x) \cdot\left(x^{\prime}-y^{\prime}\right)=$ $t(x)-\bar{t}(y) \neq 0$. So we know that $x^{\prime}-y^{\prime} \neq 0$. Now it is clear that (i), (ii) and (iii) hold if we know that $\partial_{n} t(x)=0$. The claim is that indeed $\partial_{n} t(x)=0$, for suppose not. Consider the $(n-1) \times(n-1)$ submatrix of $H_{\phi}$ given by deleting the first row and $n$-th column. It is of the type described in Lemma 1.7. Thus $\left(x_{1}-y_{1}\right) \partial_{n} t(x)=0$, and hence $\left(x_{1}-y_{1}\right)=0$. Now delete the second row and $n$-th column from $H_{\phi}$. After switching two columns, we may again apply Lemma 1.7 to obtain that $x_{2}-y_{2}=0$, and continuing in this way we find that $x^{\prime}-y^{\prime}=0$ which is a contradiction. 
It is easy now to construct $t$ and $\bar{t}$ such that (i), (ii) and (iii) of Proposition 3.1 are satisfied, and we give a simple example to demonstrate this fact. Let $t(x)=x_{1}$ and $\bar{t}(y)=y_{n}$; then (i)-(iii) are satisfied on the set $\{(x, y)$ : $\left.x_{1}=x_{n}, y_{1}=y_{n}\right\}$. Suffice it to say that there are many $t$ and $\bar{t}$ such that $\operatorname{rank}\left(H_{\phi}\right)=n-2$, and we reserve a more detailed discussion of this for a future publication preferring to move on to the main technical lemma of this chapter.

Lemma 3.2. Let $K_{1}, K_{2} \in C_{0}^{\infty}\left(\mathbb{R}^{n} \times \mathbb{R}^{n}\right)$ and let

$$
T_{j} f(x)=\int_{\mathbb{R}^{n}} f(y) K_{j}(x, y) d y \quad j=1,2 .
$$

Suppose that there is an open set $U \subset \mathbb{R}^{n}$ such that

$$
\operatorname{supp}\left(K_{2}\right) \subset U \quad \text { and } \quad K_{1} \neq 0 \text { on } \bar{U} .
$$

If there are constants $C_{1}, C_{2}$ and $C_{3}>0$ such that

$$
\begin{aligned}
& \left\|K_{2} / K_{1}\right\|_{L^{1}\left(\mathbb{R}^{n} \times \mathbb{R}^{n}\right)} \leq C_{1}, \\
& \left\|\sum_{|\alpha|=s} \mathrm{D}^{\alpha}\left(K_{2} / K_{1}\right)\right\|_{L^{2}\left(\mathbb{R}^{n} \times \mathbb{R}^{n}\right)} \leq C_{2} \quad \text { for some } s>n, \\
& \operatorname{supp}\left(K_{2}\right) \subset B_{C_{3}}(0) \times B_{C_{3}}(0) .
\end{aligned}
$$

Then

$$
\left\|T_{2}\right\| \leq C\left\|T_{1}\right\|
$$

where $C=C\left(C_{1}, C_{2}, C_{3}\right)$ is bounded once $C_{1}, C_{2}$ and $C_{3}$ are bounded.

The proof of this lemma is simple: we write $K_{2}$ as the product of $K_{1}$ and an absolutely convergent sum of compactly supported tensors via an application of the Fourier transform in the two variables $x, y$. Then $T_{2}$ is the absolutely convergent sum of operators each one being obtained from $T_{1}$ by composition with unitary operators. A full proof may be found in $[\mathbf{K}]$, and I am indebted to T. Wolff for pointing out the existence of such a lemma.

If we let $K_{1}(x, y)=e^{i \lambda \phi(x, y)} b(x, y)$ and $K_{2}(x, y)=e^{i \lambda \phi(x, y)} a(x, y)$, the 
conditions (3.2) and (3.3) in this case translate as

$$
\begin{aligned}
& \exists U \subset \mathbb{R}^{n} \text { open, } \operatorname{supp}(a) \subset U \text { and } b \neq 0 \text { on } \bar{U}, \\
& \|a / b\|_{L^{1}\left(\mathbb{R}^{n} \times \mathbb{R}^{n}\right)} \leq C_{1}, \\
& \left\|\sum_{|\alpha|=s} \mathrm{D}^{\alpha}(a / b)\right\|_{L^{2}\left(\mathbb{R}^{n} \times \mathbb{R}^{n}\right)} \leq C_{2} \quad \text { for some } s>n, \\
& \operatorname{supp}(a) \subset B_{C_{3}}(0) \times B_{C_{3}}(0) .
\end{aligned}
$$

From Proposition 3.1 and Theorem 1.8 we readily obtain the result $\left\|T_{\lambda} f\right\|_{2} \leq C \lambda^{-(n-2) / 2}\|f\|_{2}$, and although it is possible to find functions which satisfy conditions (i), (ii) and (iii) of Proposition 3.1 at a point, or on even larger varieties, it is not possible that $H_{\phi}$ should be so singular that such an estimate is sharp. This is the content of our main theorem.

Theorem 3.3. Let $T_{\lambda}$ be the oscillatory integral operator with phase function $\phi$ as above. Then

$$
\left\|T_{\lambda} f\right\|_{2} \leq C \lambda^{-(n-1) / 2}\|f\|_{2} .
$$

Proof. Let $B_{R}=\left\{x \in \mathbb{R}^{n}:|x|<R\right\}$. Suppose with out loss of generality (after perhaps a dilation) that $\operatorname{supp}(a) \subset B_{1} \times B_{1}$. Assume also (after a change of the parameter $\lambda)$ that $1 \leq t(x)-\bar{t}(y)$. Choose $\psi \in C_{0}^{\infty}\left(B_{5}\right)$ with $\psi \equiv 1$ on $B_{4}$. Consider the following operators:

$$
\begin{aligned}
& S_{\lambda}^{1} f(x)=\int_{\mathbb{R}^{n}} e^{i \lambda\left(-2 x \cdot y+t(x)|y|^{2}\right)} \psi(x) \psi(y) f(y) d y, \\
& S_{\lambda}^{2} f(x)=\int_{\mathbb{R}^{n}} e^{i \lambda\left(-2 x \cdot y+\bar{t}(x)|y|^{2}\right)} \psi(x) \psi(y) f(y) d y .
\end{aligned}
$$

We know that

$$
\left\|S_{\lambda}^{j}\right\| \lesssim \lambda^{-n / 2+1 / 4} \quad j=1,2
$$

Thus

$$
\left\|\lambda^{n / 2} S_{\lambda}^{1}\left(S_{\lambda}^{2}\right)^{*}\right\| \lesssim \lambda^{-n / 2+1 / 2}
$$

Now

$$
\lambda^{n / 2} S_{\lambda}^{1}\left(S_{\lambda}^{2}\right)^{*} f(x)=\int_{\mathbb{R}^{n}} e^{i \lambda \frac{|x-z|^{2}}{t(x)-t(z)}} b_{\lambda}(x, z) f(z) d z
$$


where

$$
\begin{aligned}
b_{\lambda}(x, z)=\lambda^{n / 2} \frac{\psi(x) \psi(z)}{(t(x)-\bar{t}(z))^{n / 2}} \int_{\mathbb{R}^{n}} e^{i \lambda|y|^{2}} \psi^{2} \\
\cdot\left(\frac{y}{(t(x)-\bar{t}(z))^{1 / 2}}+\frac{x-z}{t(x)-\bar{t}(z)}\right) d y .
\end{aligned}
$$

We wish to apply Lemma 3.2 to finish the proof; this amounts to checking $\left(3.2^{\prime}\right)$ and $\left(3.3^{\prime}\right)$ for $a$ and $b_{\lambda}$. Let $U=B_{2} \times B_{2}$, and suppose that $(x, z) \in \bar{U}$ — i.e. $|x| \leq 2,|z| \leq 2$. We apply Theorem 1.3 to the integral in (3.6) to obtain that

$$
b_{\lambda}(x, z)=\pi^{\frac{n}{2}} e^{-i \frac{n}{4} \pi} \frac{\psi(x) \psi(z)}{(t(x)-\bar{t}(z))^{n / 2}}\left(\psi^{2}\left(\frac{x-z}{t(x)-\bar{t}(z)}\right)+E(x, z)\right),
$$

where $E(x, z)$ is the first-order remainder in (1.2). Now since

$$
\left|\frac{x-z}{t(x)-\bar{t}(z)}\right| \leq 4 \quad \text { when }(x, z) \in \bar{U}
$$

then

$$
\psi^{2}\left(\frac{x-z}{t(x)-\bar{t}(z)}\right)=1 \quad \text { when }(x, z) \in \bar{U}
$$

Moreover by 1.3 ,

$$
|E(x, z)| \leq C \lambda^{-1} \sum_{|\alpha| \leq n / 2+2}\left\|\mathrm{D}^{\alpha} \psi^{2}\right\|_{2} \leq 1 / 2 \quad \text { when } \lambda \gg 1
$$

So for large $\lambda$ (depending only on $\phi),\left|b_{\lambda}(x, z)\right| \geq 1 / 2$ on $\bar{U}$. We also see from Corollary 1.5 that

$$
\left\|\mathrm{D}^{\alpha} b_{\lambda}\right\|_{\infty} \lesssim 1 \quad \text { for }|\alpha| \leq n+1
$$

then $\left(3.2^{\prime}\right)$ and $\left(3.3^{\prime}\right)$ are satisfied with $C_{1}, C_{2}$ and $C_{3} \lesssim 1$.

Again, we may not make an improvement in Theorem 3.3 as evidenced by the follwing.

Theorem 3.4. For a given amplitude function $a \not \equiv 0$, we may find $t$ and $\bar{t}$ such that the exponent of $\lambda$ in Theorem 3.3 is sharp.

Proof. For some $\tilde{x}, \tilde{y} \in \mathbb{R}^{n}, a(\tilde{x}, \tilde{y}) \neq 0$. Following Remark 2.5 we may assume that $\tilde{y}=0$ and $\tilde{x}=\left(0, \cdots, 0, \tilde{x}_{n}\right)$ for some $\tilde{x}_{n}>0$, and furthermore 
we may reduce the size of $\operatorname{supp}(a)$ so that $c^{-1} \leq x_{n}-y_{n} \leq c$ for some fixed constant $c>0$. Finally we may assume that $a(x, y) \geq 0$.

Let $t(x)=x_{n}$ and $\bar{t}(y)=y_{n}$, and choose $\tilde{f}$ to be a $C_{0}^{\infty}$ function which is unity on $\operatorname{supp}_{y}(a)$ and such that $\|\tilde{f}\|_{2}=1$. Noting that

$$
\frac{|x-y|^{2}}{x_{n}-y_{n}}=\frac{\left|x^{\prime}-y^{\prime}\right|^{2}}{x_{n}-y_{n}}+x_{n}-y_{n}
$$

take $f(y)=e^{i y_{n}} \tilde{f}(y)$. Then by (1.2) and (1.3)

$$
\begin{aligned}
\left|T_{\lambda} f(x)\right| & =\left|\iint e^{i \frac{\lambda}{x_{n}-y_{n}}\left|x^{\prime}-y^{\prime}\right|^{2}} d y^{\prime} d y_{n}\right| \\
& =\left|\int\left(\frac{i \lambda}{\pi}\right)^{-\frac{n-1}{2}} a\left(x,\left(x^{\prime}, y_{n}\right)\right) d y_{n}+\mathcal{O}\left(\lambda^{-(n-3) / 2}\right)\right|
\end{aligned}
$$

Thus $\left\|T_{\lambda} f\right\|_{2} \gtrsim \lambda^{-n / 2+1 / 2}$ as desired.

We finish this section by noting that the estimate in Theorem 3.3 may be improved when we have a better bound on one of the "factors" $S_{\lambda}^{1}$ or $S_{\lambda}^{2}$ of $T_{\lambda}$. If for example $t$ and $\bar{t}$ satisfy the hypothesis of Theorem 2.9 then in fact $\left\|T_{\lambda}\right\| \lesssim \lambda^{-n / 2}$. More precisely we have the following theorem whose proof is simply that of Theorem 3.3.

Theorem 3.5. Let $t, \bar{t}$ and $\phi$ be as above. For a given amplitude a with support contained in $B_{1} \times B_{1}$, let

$$
T_{\lambda} f(x)=\int_{\mathbb{R}^{n}} \exp ^{i \lambda \frac{|x-y|^{2}}{t(x)-t(y)}} a(x, y) f(y) d y .
$$

If $S_{\lambda}^{1}$ and $S_{\lambda}^{2}$ in (3.4) and (3.5) are such that

$$
\left\|S_{\lambda}^{1}\right\| \lesssim \lambda^{-p} \quad \text { and } \quad\left\|S_{\lambda}^{2}\right\| \lesssim \lambda^{-q}
$$

then

$$
\left\|T_{\lambda} f\right\|_{2} \lesssim \lambda^{-p-q+n / 2}\|f\|_{2}
$$

\section{Appendix.}

We finish this paper with a few concluding remarks.

In the introduction we stated the following theorem. 
Theorem 4.1. Let

$$
T_{\lambda} f(x)=\int_{\mathbb{R}^{n}} \exp \left(i \lambda \frac{|x-y|^{2}}{t(x)-\bar{t}(y)}\right) a(x, y) f(y) d y
$$

where $a \in C_{0}^{\infty}\left(\mathbb{R}^{n} \times \mathbb{R}^{n}\right)$ and $t$ and $\bar{t}$ are measurable functions defined on $\operatorname{supp}_{x}(a)$ and $\operatorname{supp}_{y}(a)$ respectively such that $1 \leq|t(x)-\bar{t}(y)| \leq 2$. Then

$$
\left\|T_{\lambda} f\right\|_{2} \leq C \lambda^{-\frac{n-2}{4}}\|f\|_{2} .
$$

Although this theorem does not appear in $[\mathbf{B}]$, some of the ideas in the proof may be found there. It is interesting to note that in spite of the non-smoothness of the phase function, $\left\|T_{\lambda}\right\| \rightarrow 0$ as $\lambda \rightarrow \infty$ when $n>2$.

Proof. For $\epsilon$ to be chosen, let

$$
\begin{aligned}
U_{j} & =\left\{x \in \operatorname{supp}_{x}(a): j \epsilon \leq t(x)<(j+1) \epsilon\right\} \\
\bar{U}_{k} & =\left\{x \in \operatorname{supp}_{y}(a): k \epsilon \leq \bar{t}(y)<(k+1) \epsilon\right\} .
\end{aligned}
$$

Letting $\chi_{j}(x)$ and $\bar{\chi}_{k}(y)$ be the characteristic functions of $U_{j}$ and $\bar{U}_{k}$ respectively, we see that

$$
\begin{aligned}
T_{\lambda} f(x)= & \sum_{j k} \int_{\mathbb{R}^{n}} \exp \left(i \lambda \frac{|x-y|^{2}}{t(x)-\bar{t}(y)}\right) \chi_{j}(x) a(x, y) \bar{\chi}_{k}(y) f(y) d y \\
= & \sum_{j k} \int_{\mathbb{R}^{n}} \exp \left(i \lambda \frac{|x-y|^{2}}{t_{j}-\bar{t}_{k}}\right) \chi_{j}(x) a(x, y) \bar{\chi}_{k}(y) f(y) d y \\
& +\int_{\mathbb{R}^{n}} \sum_{j k}\left(\exp \left(i \lambda \frac{|x-y|^{2}}{t(x)-\bar{t}(y)}\right)\right. \\
& \left.\quad-\exp \left(i \lambda \frac{|x-y|^{2}}{t_{j}-\bar{t}_{k}}\right)\right) \chi_{j}(x) a(x, y) \bar{\chi}_{k}(y) f(y) d y \\
= & T_{\lambda 1} f(x)+T_{\lambda_{2}} f(x),
\end{aligned}
$$

where $t_{j} \in[j \epsilon,(j+1) \epsilon)$ and $\bar{t}_{k} \in[k \epsilon,(k+1) \epsilon)$. When $x \in U_{j}$ and $y \in \bar{U}_{k}$ it is clear that

$$
\left|\exp \left(i \lambda \frac{|x-y|^{2}}{t(x)-\bar{t}(y)}\right)-\exp \left(i \lambda \frac{|x-y|^{2}}{t_{j}-\bar{t}_{k}}\right)\right| \lesssim \lambda \epsilon .
$$

Then by Schur's Lemma

$$
\left\|T_{\lambda 1} f\right\|_{2} \lesssim \lambda \epsilon\|f\|_{2}
$$


Now we estimate $\left\|T_{\lambda 2}\right\|$ by duality. Let $g \in L^{2}$ be such that $\|g\|_{2}=1$. First notice that the number of indices $j$ or $k$ is comparable to $\epsilon^{-1}$, and let $T_{j k}$ be the oscillatory integral operator with phase function $\exp \left(i \lambda \frac{|x-y|^{2}}{t_{j}-\bar{t}_{k}}\right)$ and amplitude $\chi_{j}(x) a(x, y) \bar{\chi}_{k}(y)$. Then

$$
\begin{aligned}
& \left|\int_{\mathbb{R}^{n}} T_{\lambda_{2}} f(x) g(x) d x\right| \\
& =\left|\sum_{j k} \int_{\mathbb{R}^{n}} \int_{\mathbb{R}^{n}} \exp \left(i \lambda \frac{|x-y|^{2}}{t_{j}-\bar{t}_{k}}\right) g(x) \chi_{j}(x) a(x, y) \bar{\chi}_{k}(y) f(y) d x d y\right| \\
& \leq \sum_{j k}\left\|g \bar{\chi}_{k}\right\|_{2}\left\|T_{j k}\left(f \chi_{j}\right)\right\|_{2} \leq \sum_{j}\left(\sum_{k}\left\|g \bar{\chi}_{k}\right\|_{2}^{2}\right)^{1 / 2}\left(\sum_{k}\left\|T_{j k}\left(f \chi_{j}\right)\right\|_{2}^{2}\right)^{1 / 2} \\
& \lesssim\|g\|_{2}\|f\|_{2} \lambda^{-n / 2} \epsilon^{-1} .
\end{aligned}
$$

So

$$
\left\|T_{\lambda 2} f\right\|_{2} \lesssim \lambda^{-n / 2} \epsilon^{-1}\|f\|_{2} .
$$

Choosing $\epsilon=\lambda^{-n / 4-1 / 2}$ in (4.2) and (4.3) yields (4.1).

Further study involves relaxing the smoothness assumption on $t$ and $\bar{t}$. One would like to drop all smoothness assumptions in order to prove pointwise convergence results in higher dimensions. The scheme in $[\mathbf{B}]$ can certainly be carried out in three space dimensions. In higher dimensions it seems more likely that one will have to study directly the operator $R_{k}$ mentioned in the introduction, without recourse to $T_{\lambda}$. Preliminary results in this direction can be found in $[\mathbf{K}]$.

\section{References}

[B] J. Bourgain, A Remark on Schrödinger Operators, Isr. J. of Math., 77 (1992), 1-16.

[C] L.Carleson, Some Analytical Problems Related to Stastical Mechanics, Euclidean Harmonic Analysis, Lecture Notes in Math., Springer-Verlag, Berlin and New York, 779 (1979), 5-45.

[DK] B. Dahlberg and C. Kenig, A Note on the Almost Everywhere Convergence of Solutions of the Schrödinger Equation, Harmonic Analysis, Lecture Notes in Math., Springer-Verlag, Berlin, 908 (1982), 205-209.

[GS] A. Greenleaf and A. Seeger, Fourier Integral Operators With Fold Singularities, preprint.

[H1] L. Hörmander, The Analysis of Linear Partial Differential Operators I, SpringerVerlag, Grund. der math. Wiss, Berlin and New York, 256 (1990). 
[H2] - Oscillatory Integrals and Multipliers on $F L^{p}$, Ark. Mat., 11 (1971), 1-11.

[K] L. Kolasa, Oscillatory Integral Operators Related to Pointwise Convergence of Schrödinger Operators, Ph.D. Thesis California Institute of Technology, 1994.

[PS] Y. Pan and C. Sogge, Oscillatory Integrals Associated to Folding Canonical Relations, Colloquium Mathematicum, 60 (1990), 413-419.

[Sj] P. Sjölin, Regularity of Solutions to the Schrödinger Equation, Duke Math. J., 55 (1987), 669-715.

[St] E.M. Stein, Harmonic Analysis, Princeton Univ. Press, Princeton N.J., 1993.

[V] L. Vega, Schrödinger Equations: Pointwise Convergence to the Initial Data, Proc. Am. Math. Soc., 102 (1988), 874-878.

Received September 16, 1994 and revised February 22, 1995. This work was supported in part by a Sloan Doctoral Dissertation Fellowship.

UNIVERSITY OF WISCONSIN-PARKSIDE

Kenosha, WI 53141-2000

E-mail address: kolasa@cs.uwp.edu

CURRENT ADDRESS:

UNIVERSITY OF TORONTO

100 St. George St.

Toronto, ON M59 1A1

CANADA

E-mail address: kolasa@math.toronto.edu 